\title{
Estudo da produção de arroz brasileira e o papel do estado Mato Grosso do Sul
}

\section{Study of Brazilian rice production and the role of Mato Grosso do Sul state}

\author{
Leandro Kenji Sato \\ Universidade Federal da Grande Dourados (UFGD) \\ E-mail: le inagaki@hotmail.com \\ OrclD: 0000-0001-6378-7881 \\ João Gilberto Mendes dos Reis \\ Universidade Paulista (UNIP) \\ E-mail: joao.reis@docente.unip.br \\ OrclD: 0000-0001-6409-2299
}

\begin{abstract}
Resumo: O arroz é considerado alimento básico para mais da metade da população mundial, sendo produzidas cerca de 480 milhões de toneladas por ano. O Brasil é um grande consumidor e produtor de arroz concentrado principalmente na região sul do país. Entretanto, nota-se uma dependência de outros estados inclusive de importação de grãos. O estado do Mato Grosso do Sul (MS), por exemplo, aumentou a sua produtividade em $965 \%$ nos últimos 40 anos, porém estranhamente houve grande redução da produção do grão no estado. Diante deste contexto, o presente trabalho tem como objetivo analisar a evolução do cultivo do arroz no país, as principais regiões produtoras e, mais especificamente, mostrar o panorama do cultivo do arroz no estado do Mato Grosso do Sul para identificar possíveis causas que levam este a não figurar como grande produtor apesar da produtividade registrada. Para esse fim foi realizado um estudo bibliográfico e estatístico descritivo. Os resultados mostraram que entre as causas para esse papel pouco relevante de MS está atrelado a baixa qualidade dos grãos produzidos, economias de escala na produção no Rio Grande do Sul, alta produção de soja e milho, baixa disponibilidade de financiamento, preço competitivo do arroz produzido no Paraguai e problemas logísticos.
\end{abstract}

Palavras-chave: Cadeias Produtivas; Orizicultura; Produtividade Agrícola.

Abstract: Rice is considered a staple food for more than half of the world population where the production is estimated at around 480 million tons per year. Brazil is a major consumer and producer of rice mainly in the south region of the country. However, there is a dependence on other states, including grain imports. The state of Mato Grosso do Sul (MS), for example, raise its productivity by $965 \%$ in the last 40 years, but strangely there was a great reduction in the production of grain in the state. Given this context, the present work aims to analyze the evolution of rice cultivation in the country, the main producing regions and, more specifically, to show the panorama of rice cultivation in the state of Mato Grosso do Sul to identify possible causes that lead this state not to appear as a major producer despite the recorded productivity. To this end, a descriptive bibliographic and statistical study was carried out. The results showed that among the causes for this irrelevant role of MS is linked to the low quality of the grains produced, economies of scale in production in Rio Grande do Sul, high production of soy and corn, low availability of financing, competitive price of rice produced in Paraguay and logistical issues.

Keywords: Agricultural Productivity; Rice Cultivation; Productive Chain.

Data de recebimento: $27 / 12 / 2018$

Data de aprovação: 10/08/2020

DOI: https://doi.org/10.30612/agrarian.v13i50.9212

\section{Introdução}

O arroz é considerado alimento básico para mais 3 milhões de pessoas (NATURE, 2014), sendo produzidas cerca de 480 milhões de toneladas de arroz beneficiado por ano. A maior parte do arroz cultivado e consumido se encontra na região asiática em que China e a Índia são responsáveis por mais de $50 \%$ do total 
da produção mundial. O cereal é de extrema importância para a segurança alimentar, já que ele fornece até $50 \%$ da oferta de calorias para grande parte da população asiática que vivem na miséria. A América Latina e a África também vêm apresentando um crescimento considerável no consumo do mesmo (MUTHAYYA et al., 2014). A cultura do arroz é realizada em diversos sistemas e ambientes de cultivo, podendo ser desde monoculturas em regiões temperadas e tropicais com sistemas de sequeiro e irrigado, até monocultura intensiva com áreas irrigadas nos trópicos onde a cultura é realizada duas ou três vezes anualmente (LABORTE et al., 2017).

O arroz possui grande variedade de uso dentro da gastronomia e está entre os principais alimentos mais consumidos pelos brasileiros. O famoso "arroz com feijão", prato típico do Brasil, está presente na mesa de quase toda a população, especialmente as classes mais carentes, por ser um alimento mais barato e fornecer energia e nutrientes necessários a realização das atividades diárias. Em 2016, o consumo foi de 32,2 $\mathrm{kg} /$ habitante (EMBRAPA, 2018a). Desse modo, é de extrema importância ações sociais e governamentais que incentivem o cultivo com a finalidade de assegurar que os níveis de oferta se mantenham.

Este estudo analisa a cadeia produtiva de arroz brasileira e o papel do estado de Mato Grosso do Sul. O motivo pela escolha do Estado ocorreu devido o cenário observado ao longo dos anos de alta produtividade (toneladas/hectare) de $965 \%$ nos últimos 40 anos. Entretanto, apesar dessa produtividade ter aumentado significativamente, observou-se também quedas significativas na produção (toneladas) e na área (hectares) destinada a cultura do cereal levando a questionar o que poderia ter acontecido a produção de arroz do Estado. O MS passou então de potencial produtor, com altas taxas de produtividade, para um dos menores produtores em termos de quantidade produzida (CONAB, 2020).

Diante desse contexto, o presente trabalho tem como objetivo analisar a evolução do cultivo do arroz do país, as principais regiões produtoras e, mais especificamente, mostrar o panorama do cultivo do arroz no Mato Grosso do Sul para tentar entender os motivos da queda tão grande da produção no estado. A divisão do estudo foi realizada de acordo com a seguinte estrutura: introdução, seguido da metodologia, resultados e discussões e finalizado com as considerações do trabalho.

\section{Material e Métodos}

Para compreender o panorama da produção do arroz brasileiro, encontrar as principais regiões produtoras e mostrar um diagnóstico do cultivo no Mato Grosso do Sul, foi realizado uma pesquisa exploratória, utilizando dados obtidos principalmente de fontes como: Companhia Nacional de Abastecimento (CONAB), Empresa Brasileira de Pesquisa Agropecuária (EMBRAPA), Instituto Brasileiro de Geografia e Estatística (IBGE), artigos encontrados em bases de dados nacionais e internacionais, como o Web of Science, Science Direct e SCieLo. Na visão de Gil (2010) a pesquisa exploratória tem como objetivo aumentar o conhecimento sobre determinado problema, tornando mais explícito e facilitando a construção de hipóteses. O planejamento da pesquisa exploratória é mais flexível, pois são considerados aspectos variados relativos ao tema estudado.

A partir dos dados obtidos, foi desenvolvido um estudo estatístico descritivo, que tem como objetivo resumir as principais informações de um determinado tipo de dados através de tabelas, gráficos ou resumos numéricos (GUIMARÃES, 2007). Os dados foram organizados por meio do uso de construção de gráficos e tabelas que permitiram evidenciar melhor visualização do cenário nacional do arroz a partir de dados secundários coletados das séries históricas de área plantada, produtividade e produção brasileira, relativas às safras 1976/77 a 2018/19 de arroz que é o período disponível na base de dados do site da Companhia Nacional de Abastecimento (CONAB).

\section{Resultados e Discussões}

\section{Produção Brasileira de Arroz}

A produção mundial de arroz em 2018 foi de 783,9 milhões de toneladas em 167 milhões de hectares plantados, consolidando uma produtividade média de $4.691 \mathrm{~kg} / \mathrm{ha}$ (FAO, 2020). A Tabela 1 apresenta os principais países produtores no ano de 2018. 
Tabela 1. Produção mundial de arroz no ano de 2018.

\begin{tabular}{ccc}
\hline Pais & Produção $(\mathbf{t})$ & $\%$ \\
\hline China & 214.078 .796 & $27,31 \%$ \\
İ́dia & 172.580 .000 & $22,01 \%$ \\
Indonésia & 83.037 .000 & $10,59 \%$ \\
Bangladesh & 56.417 .319 & $7,20 \%$ \\
Vietnam & 44.046 .250 & $5,62 \%$ \\
Tailândia & 32.192 .087 & $4,11 \%$ \\
Myanmar & 25.418 .142 & $3,24 \%$ \\
Filipinas & 19.066 .094 & $2,43 \%$ \\
Brasil & 11.749 .192 & $1,50 \%$ \\
Paquistão & 10.802 .949 & $1,38 \%$ \\
Cambódia & 10.647 .212 & $1,36 \%$ \\
Outros & 103.914 .900 & $13,26 \%$ \\
\hline Total & 783.949 .941 & $100 \%$
\end{tabular}

Fonte: Elaborada pelos autores com dados FAO, Food and Agriculture Organization of the United Nations, 2020.

Conforme a Tabela 1 é possível evidenciar que o Brasil foi o nono maior produtor do cereal e o primeiro país não asiático a aparecer na lista, produzindo 11,74 milhões (t) em 2018 , o que equivale a cerca de $1,50 \%$ da produção mundial.

Os fatores climáticos afetam diretamente a produção de arroz que explica a concentração dessa produção nas regiões do Sul do país onde o clima e as característica geomorfológicas são bastantes diferentes do restante da nação. Dentro dos principais fatores climáticos que afetam a cultura do arroz no crescimento, desenvolvimento e, consequentemente, a produtividade no Brasil, estão a temperatura do ar, a radiação solar, o nível de precipitação pluvial e a influência dos fenômenos "el niño" e "la niña" (SOCIEDADE SUL-BRASILEIRA DE ARROZ IRRIGADO, 2016)

No Brasil a produção do arroz se dá por diferentes sistemas de cultivos. A escolha pelo sistema depende dos fatores climáticos de cada região, e a produção é feita principalmente por dois métodos, o "cultivo em terras altas" ou cultivo de sequeiro, e pelo método que é mais comum no país, o cultivo do arroz irrigado (EMBRAPA, 2018b). Essa produção tem sido crescente nos últimos 40 anos ao mesmo tempo em que a área plantada tem diminuído, conforme pode ser visto na Figura 1.

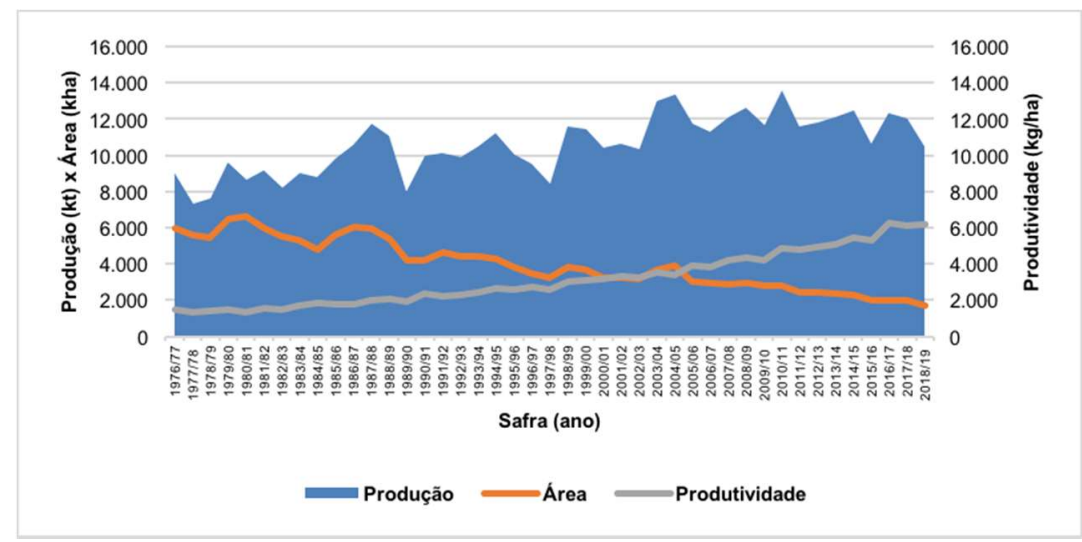

Figura 1. Evolução Produção x Área plantada x Produtividade de 1976/77 a 2018/19. Fonte: Elaborada pelos autores com dados Companhia Nacional de Abastecimento, 2020.

Conforme mostra a Figura 1, a safra de 1976/77 até a de 2018/19 houve aumento de mais de 16,57\% na produção do cereal. A safra de 1976/77 produziu aproximadamente o volume de 9 milhões (t). Durante a década de 80, embora algumas safras apresentassem quedas, pode-se observar crescimento, chegando-se a produção de 11,7 milhões (t) na safra de 1987/88. Os anos seguintes apresentaram oscilações, com altas e quedas da produção. A maior produção de arroz ocorreu na safra de 2010/11 com volume aproximado de 13,6 milhões (t). A última safra registrada foi a de 2018/19 com 10,48 milhões (t). 
Embora a produção tenha crescido ao longo dos anos, as áreas de cultivo tiveram quedas bastante expressivas. Foi observada queda de $71,59 \%$ entre as safras de 1976/77 e 2018/19. Na safra de 1976/77 foram utilizadas cerca de 5,9 milhões (ha) para a produção de arroz no país. Na metade do período acompanhado, na safra de 1996/97, houve utilização de 3,5 milhões (ha), queda de $71,5 \%$ em relação à safra de $1976 / 77$. De acordo com a última safra registrada, a safra de 2018/19, foram utilizados 1,7 milhões (ha).

A Figura 1 mostra também que com os novos níveis de produtividade do arroz no país, claramente o setor está se especializando. A safra de 2018/19 apresentou produtividade de $6.158 \mathrm{~kg} / \mathrm{ha}$, mostrando crescimento de $310 \%$ em relação à safra de 40 anos atrás, a safra de 1976/77, que teve produtividade de 1.501 $\mathrm{kg} / \mathrm{ha}$.

Considerando as médias das séries históricas, as variações entre todas as décadas foram positivas. Isto confirma novamente que o país está melhorando cada vez mais a produtividade do cereal. A maior variação ocorreu entre a média da década de 2000 a de 1990 na qual apresentou variação positiva de 49,71\% como pode ser vista na Figura 2.

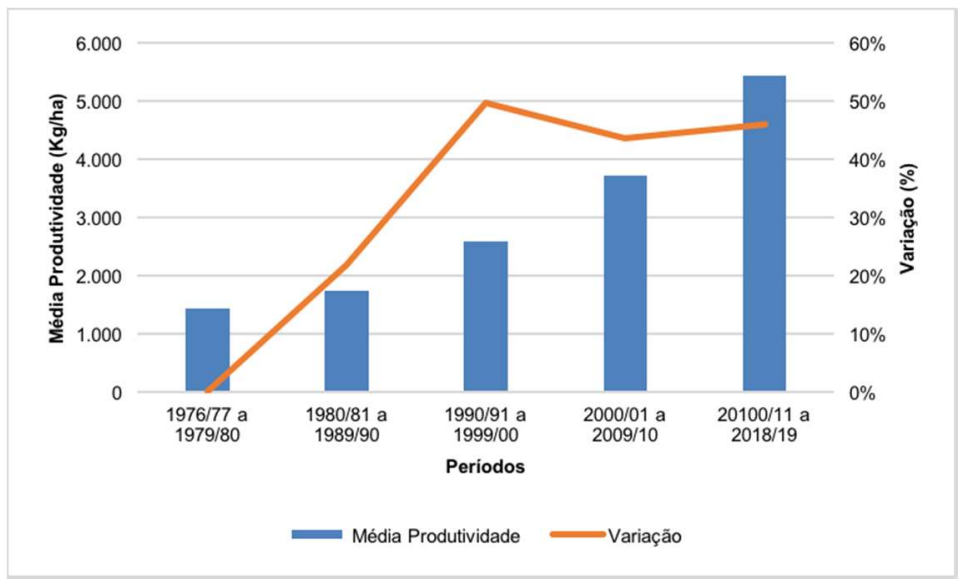

Figura 2. Média de produtividade nacional por período e variação (\%) - de 1976/77 a 2018/19. Fonte: Elaborada pelos autores com dados Companhia Nacional de Abastecimento, 2020.

Segundo as estimativas da CONAB, as estimativas da colheita de arroz nacional para a safra de 2019/20 é de 11,17 milhões $(\mathrm{t})$, alta de $6,53 \%$ em relação à safra anterior, como mostra a Tabela 2 . A região Sul é a maior produtora de arroz do Brasil, concentrando mais de $82 \%$ da produção nacional na região de acordo com a safra de 2018/19 (Figura 3). Os estados brasileiros que mais produziram nos dois últimos anos foram, respectivamente, Rio Grande de Sul, Santa Catarina, Tocantins, Mato Grosso e Maranhão (CONAB, 2020).

Tabela 2. Produção de arroz no Brasil e regiões em mil (t) *Estimativas.

\begin{tabular}{cccc}
\hline REGIÃO/UF & $\mathbf{2 0 1 8 / 1 9}$ & $\mathbf{2 0 1 9 / 2 0}$ Previsão $\left(^{*}\right)$ & Var. \% \\
\hline Norte & 940,0 & 990,5 & $5,37 \%$ \\
Nordeste & 272,0 & 309,3 & $13,71 \%$ \\
Centro-oeste & 562,4 & 594,1 & $5,63 \%$ \\
Sudeste & 48,5 & 43,0 & $-11,34 \%$ \\
Sul & $8.660,7$ & $9.231,3$ & $6,59 \%$ \\
\hline Brasil & $10.483,6$ & $11.168,2$ & $6,53 \%$
\end{tabular}

Fonte: Elaborada pelos autores com dados Companhia Nacional de Abastecimento, 2020.

\section{Regiões produtoras}

A Figura 3 mostra que as regiões Sudeste, Centro-Oeste e Nordeste tiveram quedas no decorrer dos anos. Do outro lado, as produções das regiões Norte e Sul do país cresceram, com destaque para a região Sul, com crescimento de aproximadamente $159 \%$ quando comparado a safra de 1976/77 com a de 2018/19. A 


\section{oagrartan \\ .}

produção da região Norte apesar de ter crescido, ainda continua baixa se comparada com a produção da região Sul (Figura 4).

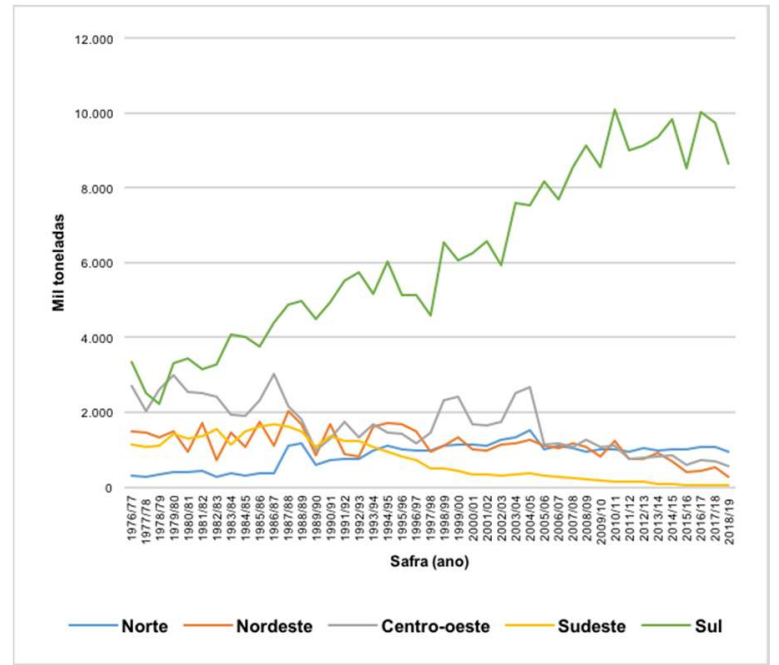

Figura 3. Produção nacional do arroz por região em milhões (t) - de 1976/77 a 2018/19. Fonte: Elaborada pelos autores com dados Companhia Nacional de Abastecimento, 2020.

Em produtividade a região Sul também domina e se destaca por sua eficiência na produção do arroz. Os estudos mostram que a produtividade da região Sul em 2018/19 foi de $7.378 \mathrm{~kg} / \mathrm{ha}$, seguido pela região Norte, Sudeste, Centro Oeste e Nordeste, com a produtividade de $4.335 \mathrm{~kg} / \mathrm{ha}, 3.666 \mathrm{~kg} / \mathrm{ha}, 3.633 \mathrm{~kg} / \mathrm{ha}$ e $1.891 \mathrm{~kg} / \mathrm{ha}$, respectivamente. Desde o início dos estudos registrados pela CONAB, a região Sul sempre apresentou a maior produtividade em relação as outras regiões (Figura 4).

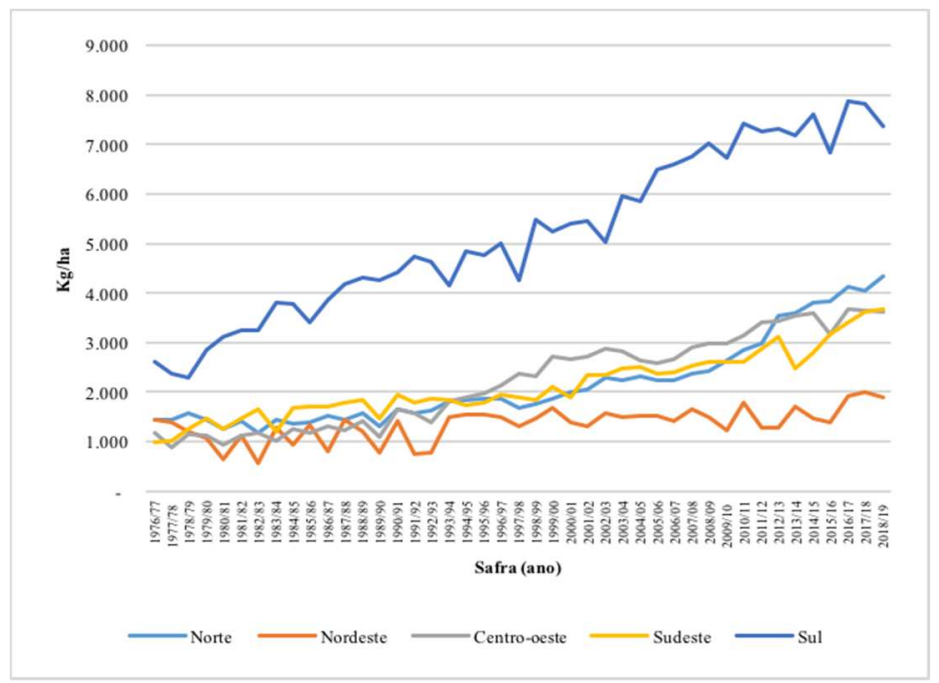

Figura 4. Produtividade do arroz por região em milhões (t) - de 1976/77 a 2018/19. Fonte: Elaborada pelos autores com dados Companhia Nacional de Abastecimento, 2020.

A Figura 5 mostra o estado do Rio Grande do Sul como o maior produtor do país de arroz desde a década de 70 . Só o estado produziu cerca de $70,48 \%$ da produção na safra de $2018 / 19$. O Rio Grande do Sul possui enorme vantagem quanto a seu clima que tem condições edafoclimáticas bastante propícias ao plantio do cereal, proporcionando altas produtividades. Além disso, o estado também possui logística bastante estratégica que facilita a comercialização do produto com praticamente todo o território brasileiro (CONAB, 2016).

O segundo maior produtor do país considerando a produção das safras de 2007/08 a 2018/19 é Santa Catarina, seguido de Tocantins, Mato Grosso e Maranhão, com 10,77\%, 5,95\%, 3,7\% e 1,24\%, respectivamente, da produção nacional na safra de 2018/19. 


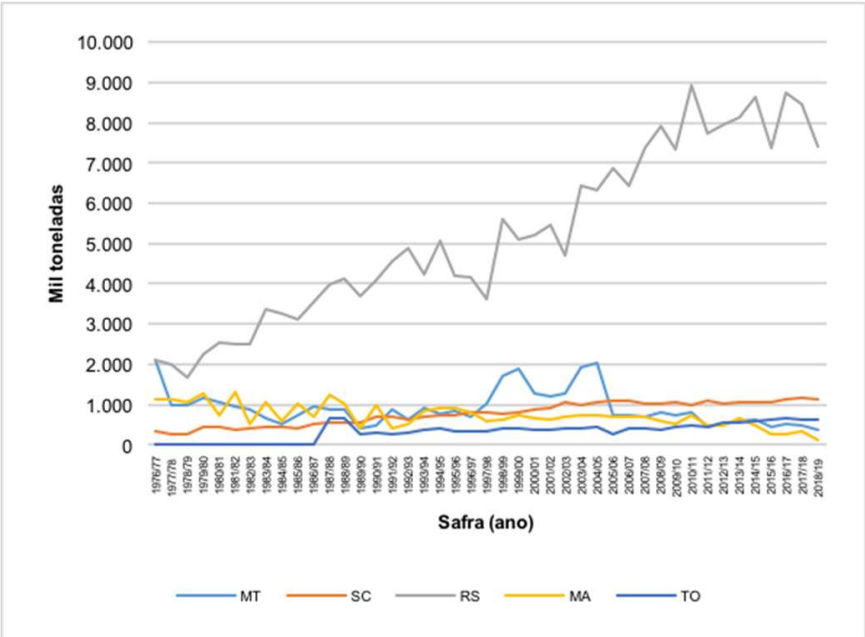

Figura 5. Principais estados produtores de 2007/08 a 2018/19. Fonte: Elaborada pelos autores com dados Companhia Nacional de Abastecimento, 2020.

\section{O arroz no Mato Grosso do Sul}

O Centro-Oeste, é a terceira região maior produtora de arroz nacional, tendo destaque o estado do Mato Grosso. O Mato Grosso do Sul, na safra de 2013/2014 produziu 95.3 toneladas, 0,78\% do total produzido nacionalmente. Esta quantidade é insuficiente para atender a demanda da região, necessitando realizar a importação de outros estados e até mesmo de outros países. As cidades de Miranda, Rio Brilhante, Dourados, Douradina, Bodoquena, Maracaju, Deodápolis, Fátima do Sul, Itaporã e Sidrolândia são as principais produtoras de arroz do estado atualmente. Entretanto, o volume é insuficiente para atender a demanda do estado sendo necessário comprar de outras regiões, principalmente o estado do Rio Grande do Sul e Paraguai (CONAB, 2016)

A Figura 6 mostra o histórico da produção das safras de arroz no estado do Mato Grosso do Sul de 1976/77 a 2018/19. Notou-se uma drástica queda ao longo dos anos de, aproximadamente, $65 \%$ da produção em relação a quantidade produzida do grão no estado. De outro lado, o estado mostrou-se bastante competente, obtendo aumento de $965 \%$ na produtividade $(\mathrm{kg} / \mathrm{ha})$.

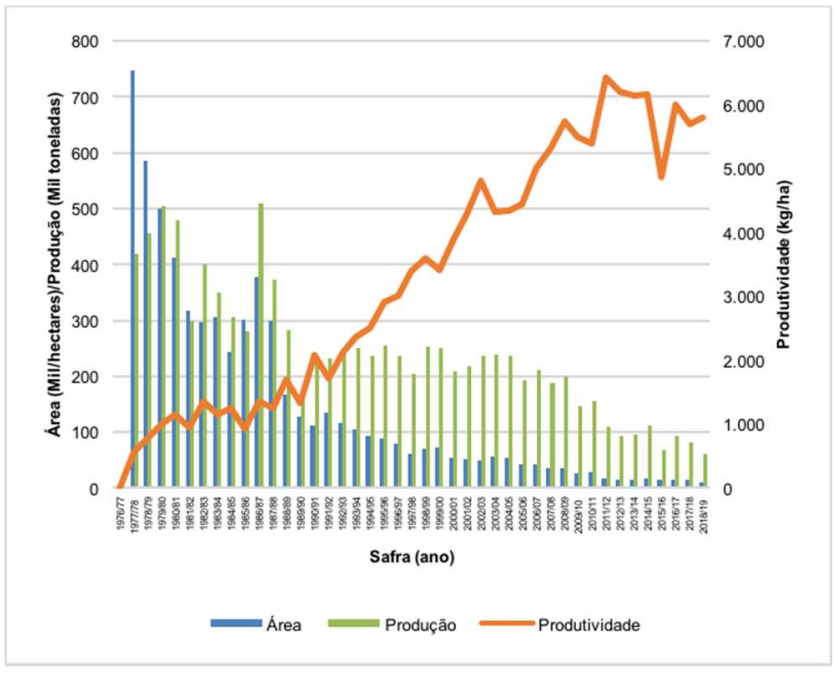

Figura 6. Histórico da produção, área e produtividade de arroz no Mato Grosso do Sul 1676/77 a 2018/19. Fonte: Elaborada pelos autores com dados Companhia Nacional de Abastecimento, 2020.

A safra de 2011/12 o estado teve produtividade de aproximadamente $6.420 \mathrm{~kg} / \mathrm{ha}$, obtendo o sétimo maior índice de produtividade do país, $85,56 \%$ acima da média nacional que foi de $3.458 \mathrm{~kg} / \mathrm{ha}$ na mesma safra 
(CONAB, 2020). Isso mostra que o estado se especializou, teve maior acesso e investiu em novas tecnologias na produção. Outro motivo para o aumento da produtividade foi a consequência da substituição do cultivo do arroz de sequeiro pela produção de arroz irrigado ao longo do tempo (PORTALETE et al., 2013). Entretanto, o estudo verificou que isso não significou um movimento da produção no Estado, explicado pela presença maciça da produção de soja na região e os ganhos de escala de outros estados como Rio Grande do Sul.

As dificuldades encontradas para explicar a queda da produção do arroz em MS foram as seguintes:

- O arroz de terras altas produz grãos de baixa qualidade, gerando elevado índice de grãos quebrados. Além disso, o clima no sistema de terras altas é outro aspecto que prejudica a produção do arroz pois favorece mais o cultivo da soja;

- A orizicultura da região sul possui alta competitividade, especialmente o estado do Rio Grande do Sul. A alta competitividade se dá por diversos motivos, entre eles, produção de boa qualidade, grande incentivo por parte do governo, incluindo alíquotas de ICMS mais baratas e redução de fretes para as outras regiões brasileiras;

- A competitividade do milho e da soja é superior à do arroz;

- Quase não há financiamentos para lavouras que pretendem cultivar arroz irrigado;

- Desvantagem com o arroz que é importado do Paraguai quanto aos custos de produção da lavoura, sendo este, aproximadamente $63 \%$ mais baixo.

- Logística deficiente em relação a infraestrutura de secagem e armazenamento devido à falta de empresas públicas e privadas que prestem este serviço e a falta de armazéns dentro das propriedades. Deste modo, os produtores são obrigados a enviar a sua produção para as indústrias logo após colher (PORTALETE et al. 2013).

\section{Conclusão}

O trabalho permitiu estabelecer um panorama da produção brasileira de arroz e analisar o declínio da produção de arroz no estado de Mato Grosso do Sul, área de influência desse estudo. A contribuição é um estudo exploratório que pode permitir aos tomadores de decisão estabelecer passos futuros no desenvolvimento desta cadeia produtiva no país e na região.

Notou-se que a Região Sul, especialmente o estado do Rio Grande do Sul, teve destaque na produção de arroz nos últimos 40 anos. O estado aumentou a sua produção e produtividade, fato este que mostra o quanto se especializou. Com produto extremamente competitivo, tanto em questões de preço, região propícia de cultivo, qualidade do produto, logística, entre outros, dificultou a ascensão da produção de outros estados.

O estado Mato Grosso do Sul é uma das regiões que sofreu as consequências de tal conjuntura. Com a queda da produção, o estado se tornou deficiente na produção de arroz e precisa comprar o produto do Rio Grande do Sul e até mesmo do Paraguai, país vizinho com que faz fronteira. Embora a produção tenha tido quedas, a produtividade cresceu expressivamente, o que mostra oportunidades para a orizicultura dentro do estado.

Como sugestão para trabalhos futuros, recomenda-se encontrar os produtores do Mato Grosso do Sul e descobrir os reais motivos da queda da orizicultura no estado, diagnosticar quais as dificuldades encontradas na produção do cereal e o porquê da desmotivação no cultivo da mesma. Ademais, sugere-se também estudar os principais produtos cultivados no Mato Grosso do Sul para entender a cadeia produtiva do estado e identificar as razões de outras culturas serem mais viáveis que a orizicultura.

\section{Referências}

CONAB. Acompanhamento da Safra Brasileira de Grãos. Brasília: Conab, v. 2, n. 12, set. 2016.

CONAB. "Séries Históricas de Área Plantada, Produtividade e Produção, relativas às Safras 1976/77 a 2018/19 de Grãos". Disponível em: https://www.conab.gov.br/info-agro/safras/serie-historica-dassafras?start=10. Acesso em: 28 julho 2020.

CONAB. Acompanhamento da Safra Brasileira de Grãos. Brasília: Conab, 2016. Disponível em: https://www.conab.gov.br/OlalaCMS/uploads/arquivos/17 $04 \quad 12 \quad 1408 \quad 06$ relatorio safra graos rn 2017 7o lev.pdf. Acesso em: 10 maio 2018. 
EMBRAPA. "Dados de conjuntura da produção de arroz (Oryza sativa L.) no Brasil (1985-2013)". Disponível em: http://www.cnpaf.embrapa.br/socioeconomia/index.htm. Acesso em: 02 abr. 2018a.

EMBRAPA. "Sistemas de produção EMBRAPA: Cultivo do arroz irrigado no Brasil". Disponível em: https://www.spo.cnptia.embrapa.br/conteudo?p p id=conteudoportlet WAR sistemasdeproducaolf6 1ga1cep ortlet\&p $p$ lifecycle $=0 \& p \quad p$ state $=$ normal\&p $p$ mode $=v i e w \& p \quad p$ col $i d=$ column $1 \& p \quad p$ col count $=1 \& p \quad r \quad p-$ 76293187 sistemaProducaold=5101\&p r p -996514994 topicold=5515. Acesso em: 01 abril $2018 \mathrm{~b}$.

FAO, Food and Agriculture Organization of the United Nations. "Statistical Databases: Agriculture, 2020". Disponível em: http://faostat3.fao.org. Acesso em 01 agosto 2020.

GIL, A.C. Como elaborar projetos de pesquisa. São Paulo: Atlas, 2010.

GUIMARÃES, P. R. B. Métodos Quantitativos Estatísticos. Curitiba: IESDE Brasil S.A, 2008.

LABORTE, A.G., GUTIERREZ, M. A., BALANZA, J. G., SAITO, K., ZWART, S. J., BOSCHETTI, M. B., MURTY, M.V.R. 2017. "RiceAtlas, a spatial database of global rice calendars and production". Scientific Data 4 (maio): 170074. https://doi.org/10.1038/sdata.2017.74.

MUTHAYYA, S., SUGIMOTO, J. D., MONTGOMERY, S., MABERLY, G. F. “An Overview of Global Rice Production, Supply, Trade, and Consumption: Global Rice Production, Consumption, and Trade". Annals of the New York Academy of Sciences 1324 (1): 7-14, 2014. https://doi.org/10.1111/nyas.12540.

NATURE. Nature outlook: Rice. Nature, 2014. Disponível em: https://www.nature.com/articles/514S49a.pdf. Acesso em: 05 setembro 2018.

PORTALETE, L. C., NETO, A. R., AZAMBUJA, D., FERREIRA, C. M. F. "Caracterização e Diagnóstico da Cadeia Produtiva do Arroz no Estado de Mato Grosso do Sul”. Brasília: Embrapa, 2013. Disponível em: https://ainfo.cnptia.embrapa.br/digital/bitstream/item/99148/1/manualilustrado-11.pdf. Acesso em: 13 maio 2018.

SOCIEDADE SUL-BRASILEIRA DE ARROZ IRRIGADO. "Arroz irrigado: recomendações técnicas da pesquisa para o sul do Brasil”. Pelotas: SOSBAI, 2016. Disponível em: http://www.sosbai.com.br/docs/Boletim RT 2016.pdf. Acesso em :15 maio 2018. 